\title{
Nearly Frobenius theory and semisimplicity of bimodules
}

\author{
Dalia Artenstein, Ana González, Gustavo Mata.
}

July 29, 2019

\begin{abstract}
In the first part of this article we prove that one of the conditions required in the original definition of nearly Frobenius algebra, the coassociativity, is redundant. Also, we determine the Frobenius dimension of the product and tensor product of two nearly Frobenius algebras from the Frobenius dimension of each of them. We apply these results to semisimple algebras.

In the second part we introduce the notion of normalized nearly Frobenius algebra. We prove a series of equivalences: the concept of normalized nearly Frobenius algebra is equivalent to the concept of separable algebra, equivalent to the fact that the algebra is projective as a bimodule on itself and, finally, equivalent to the category of bimodules is semisimple. Also, we relate these concepts with the property of semisimplicity of the category of modules over the algebra.
\end{abstract}

Keywords: nearly Frobenius algebras, separable algebra, semisimple bimodule category, normalized coproduct.

MSC: 16W99

\section{Introduction}

The concept of nearly Frobenius algebra is motivated by the result proved in 4, which states that: the homology of the free loop space $H^{*}(L M)$ has the structure of a Frobenius algebra without counit. These objects were studied in [7] and their algebraic properties were developed in 2], in particular the possible nearly Frobenius structures in gentle algebras were described.

In the framework of differential graded algebras, Abbaspour considers in [1] nearly Frobenius algebras that he calls open Frobenius algebras. He proves that if $A$ is a symmetric open Frobenius algebra of degree $m$, then $H H_{*}(A, A)[m]$ is an open Frobenius algebra, where the product at the chain level is given by

$$
a_{0}\left[a_{1}, \ldots, a_{n}\right] \circ b_{0}\left[b_{1}, \ldots, b_{m}\right]= \begin{cases}0 & \text { if } n>0 \\ a_{0}^{\prime \prime} a_{0}^{\prime} b_{0}\left[b_{1}, \ldots, b_{m}\right] & \end{cases}
$$

and the coproduct is given by

$$
\Delta\left(a_{0}\left[a_{1}, \ldots, a_{n}\right]\right)=\sum_{\left(a_{0}\right), 0 \leq i \leq n}\left(a_{0}^{\prime}\left[a_{1}, \ldots, a_{i-1}, a_{i}\right]\right) \otimes\left(a_{0}^{\prime \prime}\left[a_{i+1}, \ldots, a_{n}\right]\right) .
$$

In this work we prove that one of the conditions required in the original definition of nearly Frobenius algebra, the coassociativity, is redundant. Also, we determine the Frobenius dimension of the product and tensor product of two nearly Frobenius algebras from the Frobenius dimension of each of them. This applies to the definition of Frobenius algebras too. 
In the second part we introduce the notion of normalized nearly Frobenius algebras, we prove that cartesian and tensor product of normalized nearly Frobenius algebras is also a normalized nearly Frobenius algebra. Later, we prove that the concept of normalized nearly Frobenius algebra is equivalent to the concept of separable algebra and equivalent in turn to algebra having Hochschild cohomological dimension zero. We give some applications of these results, for example that the matrix algebra is a normalized nearly Frobenius algebra, therefore is separable. If we consider the category of bimodules over a nearly Frobenius algebra, we prove that the normalized condition over the nearly Frobenius algebra is equivalent that the bimodule category is semisimple.

The work finish relating the concepts described above with the semisimplicity property of the module category on the nearly Frobenius algebra. Although the conclusions presented in the applications are already known the techniques to prove them are originals.

\section{Nearly Frobenius algebras}

In one of the definitions of Frobenius algebras it is required that the algebra $A$ admits a coalgebra structure $(A, \Delta, \varepsilon)$ where the coproduct $\Delta$ is a morphism of $A$-bimodules. In the next result we prove that the coassociativity condition is redundant.

Proposition 1. Let $A$ be a Frobenius algebra, then the coassociativity condition is a consequence of the A-bimodule morphism condition of $\Delta$, and the unit axiom.

Proof. In the next diagram we illustrate this affirmation.

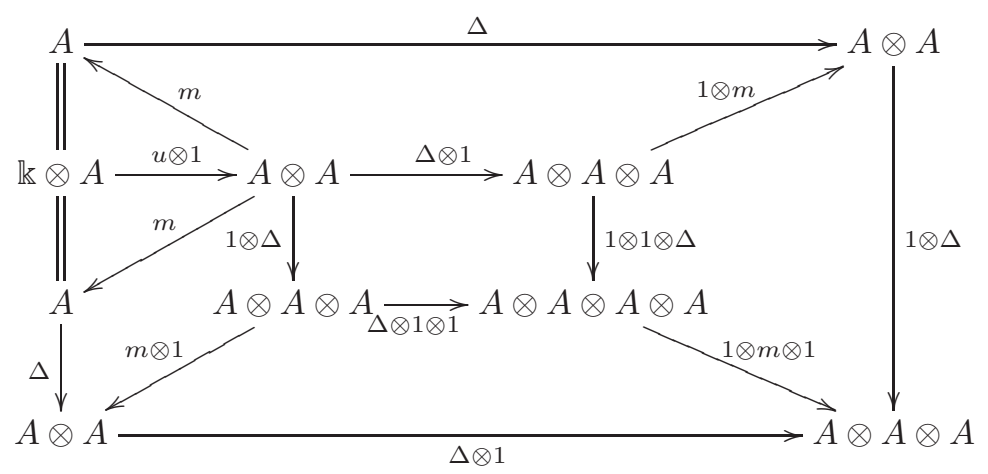

All the internal diagrams commute as a consequence of the $A$-bimodule condition, the unit axiom and the natural decomposition of the morphism $\Delta \otimes \Delta$; then the external diagram commutes too.

The previous result allows us to give the next alternative definition of nearly Frobenius algebras.

Definition 2. An algebra $A$ is a nearly Frobenius algebra if it admits a linear map $\Delta: A \rightarrow A \otimes A$ such that
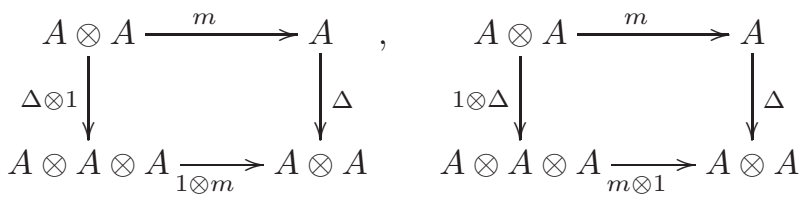

commute. 
Definition 3. The Frobenius space associated to an algebra $A$ is the vector space of all the possible coproducts $\Delta$ that make it into a nearly Frobenius algebra $(\mathcal{E})$, see [Q]. Its dimension over $\mathbb{k}$ is called the Frobenius dimension of $A$, that is,

$$
\text { Frobdim } A=\operatorname{dim}_{\mathbb{k}} \mathcal{E} .
$$

Definition 4. Let $\left(A, \Delta_{A}\right)$ and $\left(B, \Delta_{B}\right)$ be two nearly Frobenius algebras. A homomorphism $f: A \rightarrow B$ is a nearly Frobenius homomorphism if it is a morphism of algebras and the following diagram commutes.

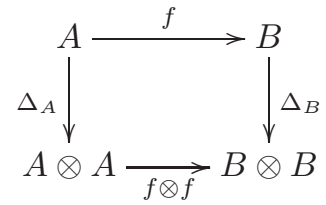

If $f$ is bijective then $f$ is said to be an isomorphism between $A$ and $B$.

Notation: nFrob is the category of nearly Frobenius algebras.

Theorem 5. Let $\left(A, \Delta_{A}\right)$ be a nearly Frobenius algebra, $B$ an algebra and $f: A \rightarrow B$ an isomorphism of algebras. Then $B$ admits a nearly Frobenius structure defined as

$$
\Delta_{B}=(f \otimes f) \circ \Delta_{A} \circ f^{-1} .
$$

In particular Frobdim $A=$ Frobdim $B$.

Proof. We need to check that $\Delta_{B}$ is a $B$-bimodule morphism. That is,

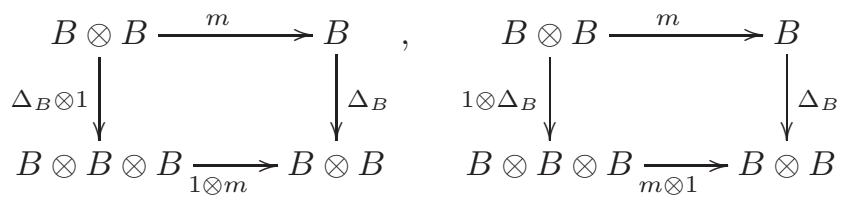

commute. To prove this we only need to see that the dotted face of the next cube commutes.

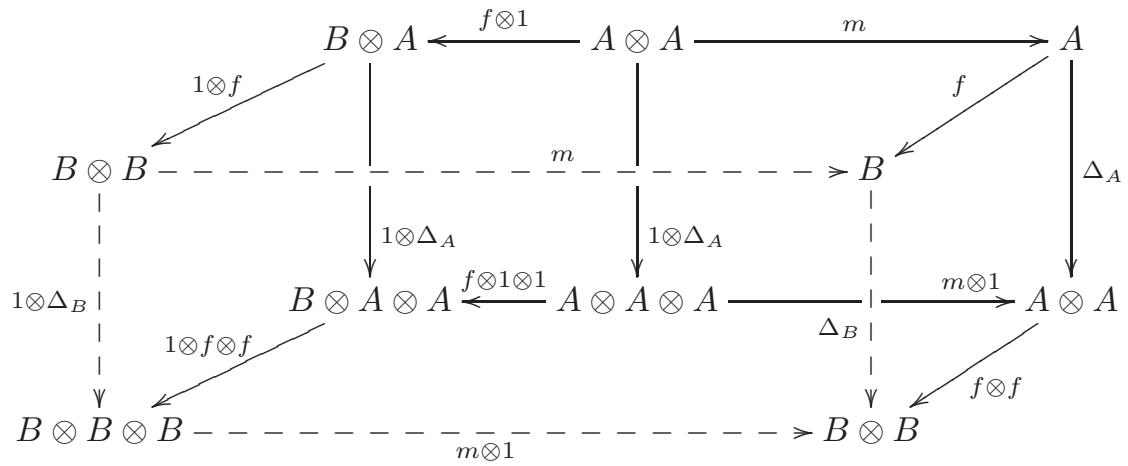

Since $f$ is an isomorphism of algebras and $\Delta_{A}$ is a nearly Frobenius coproduct in $A$ all the other faces commute and then the dotted face commutes.

Remark 6. Assume that $A_{1}$ and $A_{2}$ are $\mathbb{k}$-algebras. The product of the algebras $A_{1}$ and $A_{2}$ is the algebra $A=A_{1} \times A_{2}$ with the addition and the multiplication given by the formulas 
$\left(a_{1}, a_{2}\right)+\left(b_{1}, b_{2}\right)=\left(a_{1}+b_{1}, a_{2}+b_{2}\right)$ and $\left(a_{1}, a_{2}\right)\left(b_{1}, b_{2}\right)=\left(a_{1} b_{1}, a_{2} b_{2}\right)$, where $a_{1}, b_{1} \in A_{1}$ and $a_{2}$, $b_{2} \in A_{2}$. The identity of $A$ is the element $1=\left(1_{A_{1}}, 1_{A_{2}}\right)=e_{1}+e_{2} \in A_{1} \times A_{2}$, where $e_{1}=\left(1_{A_{1}}, 0\right)$ and $e_{2}=\left(0,1_{A_{2}}\right)$. If $\left(A_{1}, \Delta_{1}\right)$ and $\left(A_{2}, \Delta_{2}\right)$ are nearly Frobenius algebras then $A$ admits a natural structure of Nearly Frobenius algebra. In the next paragraph we describe this structure.

First, we define $\Delta\left(e_{1}\right)=\sum\left(a_{1}, 0\right) \otimes\left(a_{2}, 0\right)$, where $\Delta_{1}\left(1_{A_{1}}\right)=\sum a_{1} \otimes a_{2}$ and $\Delta\left(e_{2}\right)=\sum\left(0, b_{1}\right) \otimes$ $\left(0, b_{2}\right)$, where $\Delta_{2}\left(1_{A_{2}}\right)=\sum b_{1} \otimes b_{2}$. Then

$$
\Delta(1)=\sum\left(a_{1}, 0\right) \otimes\left(a_{2}, 0\right)+\sum\left(0, b_{1}\right) \otimes\left(0, b_{2}\right) \in A \otimes A .
$$

To prove that this defines a bimodule morphism it is necessary to guarantee that $\Delta(1)$ satisfies that

$$
(c \otimes 1) \Delta(1)=\Delta(1)(1 \otimes c), \forall c \in A .
$$

Denote $c=\left(c_{1}, c_{2}\right) \in A$, then

$$
\begin{aligned}
(c \otimes 1) \Delta(1) & =\left(c_{1}, c_{2}\right) \otimes(1,1)\left[\sum\left(a_{1}, 0\right) \otimes\left(a_{2}, 0\right)+\sum\left(0, b_{1}\right) \otimes\left(0, b_{2}\right)\right] \\
& =\sum\left(\left(c_{1}, c_{2}\right) \otimes(1,1)\right)\left(\left(a_{1}, 0\right) \otimes\left(a_{2}, 0\right)\right)+\sum\left(\left(c_{1}, c_{2}\right) \otimes(1,1)\right)\left(\left(0, b_{1}\right) \otimes\left(0, b_{2}\right)\right) \\
& =\sum\left(c_{1} a_{1}, 0\right) \otimes\left(a_{2}, 0\right)+\sum\left(0, c_{2} b_{1}\right) \otimes\left(0, b_{2}\right) .
\end{aligned}
$$

On the other hand

$$
\begin{aligned}
\Delta(1)(1 \otimes c) & =\left[\sum\left(a_{1}, 0\right) \otimes\left(a_{2}, 0\right)+\sum\left(0, b_{1}\right) \otimes\left(0, b_{2}\right)\right]\left((1,1) \otimes\left(c_{1}, c_{2}\right)\right) \\
& =\sum\left(\left(a_{1}, 0\right) \otimes\left(a_{2}, 0\right)\right)\left((1,1) \otimes\left(c_{1}, c_{2}\right)\right)+\sum\left(\left(0, b_{1}\right) \otimes\left(0, b_{2}\right)\right)\left((1,1) \otimes\left(c_{1}, c_{2}\right)\right) \\
& =\sum\left(a_{1}, 0\right) \otimes\left(a_{2} c_{1}, 0\right)+\sum\left(0, b_{1}\right) \otimes\left(0, b_{2} c_{2}\right) .
\end{aligned}
$$

Remember that $\Delta_{A_{1}}$ and $\Delta_{A_{2}}$ are bimodule morphisms, then

$$
\left(c_{1} \otimes 1\right) \Delta_{A_{1}}\left(1_{A_{1}}\right)=\sum c_{1} a_{1} \otimes a_{2}=\sum a_{1} \otimes a_{2} c_{1}=\Delta_{A_{1}}\left(1_{A_{2}}\right)\left(1 \otimes c_{1}\right)
$$

and

$$
\left(c_{2} \otimes 1\right) \Delta_{A_{2}}\left(1_{A_{2}}\right)=\sum c_{2} b_{1} \otimes b_{2}=\sum b_{1} \otimes b_{2} c_{2}=\Delta_{A_{2}}\left(1_{A_{2}}\right)\left(1 \otimes c_{2}\right)
$$

This proves that $(c \otimes 1) \Delta(1)=\Delta(1)(1 \otimes c)$. Then $A$ is a nearly Frobenius algebra.

Remark 7. Similarly, we can consider the tensor product $A \otimes B$ of the $\mathbb{k}$-algebras $A$ and $B$. As before, we can define a nearly Frobenius coproduct on $A \otimes B$. In this case we take the transposition map $\tau:(A \otimes A) \otimes(B \otimes B) \rightarrow(A \otimes B) \otimes(A \otimes B)$ and the coproduct on $A$ and $B$ to define the coproduct on $A \otimes B$ as follows

$$
\Delta:=\tau \circ \Delta_{A} \otimes \Delta_{B}: A \otimes B \rightarrow(A \otimes B) \otimes(A \otimes B) .
$$

Since all the maps are linear, the map $\Delta$ is linear too. We will test only one of the two necessary 
conditions to guarantee that it is bimodule morphism, the other one is analogous.

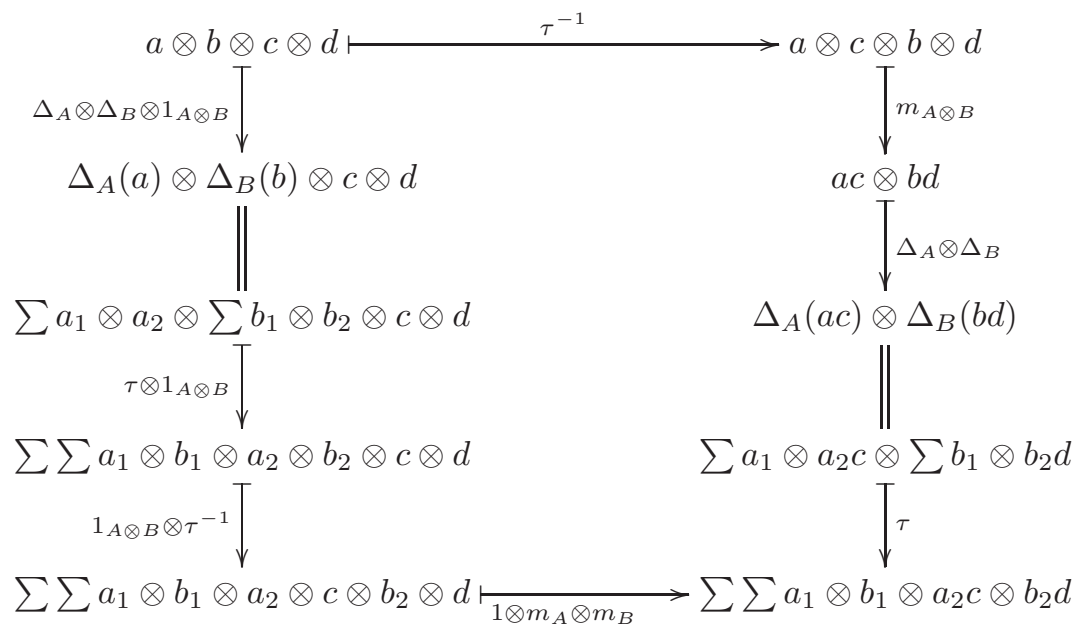

Proposition 8. Consider $A$ and $B$ two $\mathbb{k}$-algebras, then the following isomorphisms of vector spaces hold:

1. $\mathcal{E}_{A \times B} \cong \mathcal{E}_{A} \times \mathcal{E}_{B}$. In particular $\operatorname{Frobdim}(A \times B)=\operatorname{Frobdim}(A)+\operatorname{Frobdim}(B)$.

2. $\mathcal{E}_{A \otimes B} \cong \mathcal{E}_{A} \otimes \mathcal{E}_{B}$. Therefore $\operatorname{Frobdim}(A \otimes B)=\operatorname{Frobdim}(A)$. $\operatorname{Frobdim}(B)$.

Proof. In Remark 6 we saw that there exist natural inclusions of $\mathcal{E}_{A} \times \mathcal{E}_{B}$ in $\mathcal{E}_{A \times B}$ and, in Remark 7] of $\mathcal{E}_{A} \otimes \mathcal{E}_{B}$ in $\mathcal{E}_{A \otimes B}$.

To finish the proof it is necessary to check that the maps are surjective.

1. We note the unit of $A \times B$ as $1=e_{1}+e_{2}$, where $e_{1}=\left(1_{A}, 0\right)$ and $e_{2}=\left(0,1_{B}\right)$. Let's take $\Delta \in \mathcal{E}_{A \times B}$ and express $\Delta(1)$ as follows:

$$
\Delta(1)=\sum_{i, j}\left(\eta_{i}, \xi_{i}\right) \otimes\left(\rho_{j}, \nu_{j}\right)
$$

with $\eta_{i}, \rho_{j} \in A$ and $\xi_{i}, \nu_{j} \in B$ for all $i, j$. Since $\Delta$ is a bimodule morphism we can prove that $\Delta\left(e_{1}\right)=\sum_{i, j}\left(\eta_{i}, 0\right) \otimes\left(\rho_{j}, 0\right)$ and, in a similar way, that $\Delta\left(e_{2}\right)=\sum_{i, j}\left(0, \xi_{i}\right) \otimes\left(0, \nu_{j}\right)$. Then, we conclude that the coproduct has the expression

$$
\Delta(1)=\Delta\left(e_{1}\right)+\Delta\left(e_{2}\right)=\sum_{i, j}\left(\eta_{i}, 0\right) \otimes\left(\rho_{j}, 0\right)+\sum_{i, j}\left(0, \xi_{i}\right) \otimes\left(0, \nu_{j}\right) .
$$

This allows us to define $\Delta_{A}\left(1_{A}\right)=\sum_{i, j} \eta_{i} \otimes \rho_{j}$ and $\Delta\left(1_{B}\right)=\sum_{i, j} \xi_{i} \otimes \nu_{j}$. Using again that $\Delta$ is a bimodule morphism, we deduce that $\Delta_{A}$ and $\Delta_{B}$ are also bimodule morphisms, then $\left(A, \Delta_{A}\right)$ and $\left(B, \Delta_{B}\right)$ are nearly Frobenius algebras. In particular $\Delta=\iota \circ\left(\Delta_{A}+\Delta_{B}\right)$.

2. Consider $\Delta \in \mathcal{E}_{A \otimes B}$ and $\left\{x_{i}\right\},\left\{y_{j}\right\}$ bases of $A$ and $B$ respectively, where $x_{1}=1_{A}$ and $y_{1}=1_{B}$. Then

$$
\Delta\left(1_{A} \otimes 1_{B}\right)=\sum_{i, j, k, l} a_{i} b_{j} c_{k} d_{l} x_{i} \otimes y_{j} \otimes x_{k} \otimes y_{l}, \quad \text { where } a_{i}, b_{j}, c_{k}, d_{l} \in \mathbb{k} .
$$

Using that $\Delta$ is bimodule morphism we have that

$$
\Delta\left(x \otimes 1_{B}\right)=\sum_{i, j, k, l} a_{i} b_{j} c_{k} d_{l} x x_{i} \otimes y_{j} \otimes x_{k} \otimes y_{l}=\sum_{i, j, k, l} a_{i} b_{j} c_{k} d_{l} x_{i} \otimes y_{j} \otimes x_{k} x \otimes y_{l} .
$$


As $y_{j}=y_{l}=1_{B}$ when $j=l=1$ we can define

$$
\Delta_{A}\left(1_{A}\right)=\sum_{i, k} a_{i} c_{k} x_{i} \otimes x_{k}
$$

analogously we can define

$$
\Delta_{B}\left(1_{B}\right)=\sum_{j, l} b_{j} d_{l} y_{j} \otimes y_{l}
$$

Note that with these definitions the coproduct $\Delta$ is

$$
\Delta=(1 \otimes \tau \otimes 1) \circ\left(\Delta_{A} \otimes \Delta_{B}\right) .
$$

The next corollary is a consequence of Theorem 5 and Proposition 8

Corollary 9. If $A$ is a semisimple algebra over an algebraically closed field $\mathbb{k}$, then it is possible to determine completely its Frobenius dimension.

Proof. By the Artin-Wedderburn Theorem we have that $A \cong M_{n_{1}}(\mathbb{k}) \times M_{n_{2}}(\mathbb{k}) \times \cdots \times M_{n_{r}}(\mathbb{k})$. Then

$$
\operatorname{Frobdim}(A)=\operatorname{Frobdim}\left(M_{n_{1}}(\mathbb{k}) \times M_{n_{2}}(\mathbb{k}) \times \cdots \times M_{n_{r}}(\mathbb{k})\right)=\sum_{i=1}^{r} \operatorname{Frobdim}\left(M_{n_{i}}(\mathbb{k})\right)=\sum_{i=1}^{r} n_{i}^{2} .
$$

Finally

$$
\operatorname{Frobdim}(A)=\sum_{i=1}^{r} n_{i}^{2}
$$

Corollary 10. Let $G$ be a finite group. If char $(\mathbb{k})$ does not divide the order of $G$ and $\mathbb{k}$ is an algebraically closed field, then it is possible to determine completely the Frobenius dimension of $\mathbb{k} G$.

Proof. Applying Maschke's theorem we have that $\mathbb{k} G$ is a semisimple algebra then, by the previous corollary, it is possible to determine completely its Frobenius dimension.

In the next results we are going to use an example presented in [2, which has a small error in its calculation. We shall now present the result quoted and its correction.

Let $G$ be a cyclic finite group of order $n$ and the group algebra $\mathbb{k} G$, with the natural basis $\left\{g^{i}: i=1, \ldots, n\right\}$. This algebra is a nearly Frobenius algebra. Moreover, we can determine all the nearly Frobenius structures that it admits.

Using the bimodule condition of the coproduct, we can prove that a basis of the Frobenius space is

$$
\mathcal{B}=\left\{\Delta_{k}: \mathbb{k} G \rightarrow \mathbb{k} G \otimes \mathbb{k} G: k \in 1, \ldots, n\right\},
$$

where $\Delta_{1}(1)=\sum_{i=1}^{n} g^{i} \otimes g^{n+1-i}$ and $\Delta_{k}(1)=\sum_{i=1}^{k-1} g^{i} \otimes g^{k-i}+\sum_{i=k}^{n} g^{i} \otimes g^{n+k-i}$ for $k=2, \ldots, n$.

In particular, we have that

$$
\operatorname{Frobdim}(\mathbb{k} G)=|G| \text {. }
$$


The general expression of any nearly Frobenius coproduct in the unit is

$$
\Delta(1)=a_{1} \sum_{i=1}^{n} g^{i} \otimes g^{n+1-i}+\sum_{k=2}^{n} a_{k}\left(\sum_{i=1}^{k-1} g^{i} \otimes g^{k-i}+\sum_{i=k}^{n} g^{i} \otimes g^{n+k-i}\right),
$$

where $a_{i} \in \mathbb{k}$ for $i=1, \ldots, n$.

Corollary 11. If $G$ is a finite abelian group, then it is possible to determine Frobdim( $(\mathbb{k} G)$.

Proof. If $G$ is a finite abelian group, then $G=G_{1} \oplus G_{2} \oplus \cdots \oplus G_{p}$, where $G_{i}$ is a finite cyclic group for $i \in\{1, \ldots, p\}$. The group algebra $\mathbb{k} G$ is isomorphic, as a $\mathbb{k}$-algebra, to $\mathbb{k} G_{1} \otimes \mathbb{k} G_{2} \otimes \cdots \otimes \mathbb{k} G_{p}$. Therefore, applying Theorem 5 and Proposition 8

$$
\operatorname{Frobdim}(\mathbb{k} G)=\operatorname{Frobdim}\left(\mathbb{k} G_{1} \otimes \mathbb{k} G_{2} \otimes \cdots \otimes \mathbb{k} G_{p}\right)=\prod_{i=1}^{p} \operatorname{Frobdim}\left(\mathbb{k} G_{i}\right)=\prod_{i=1}^{p}\left|G_{i}\right| .
$$

Finally,

$$
\operatorname{Frobdim}(\mathbb{k} G)=\prod_{i=1}^{p}\left|G_{i}\right|
$$

Examples 2.1. We illustrate the results given in Proposition 8 with a couple of examples.

1. Let's consider the cyclic groups $G$ and $H$ where $|G|=2$ and $|H|=3$ and their corresponding group algebras $A_{1}=\mathbb{k} G, A_{2}=\mathbb{k} H$. Then, by Proposition 8, 1., $B=A_{1} \times A_{2}$ is a nearly Frobenius algebra of Frobenius dimension 5.

$$
\mathcal{E}_{A_{1}}=\operatorname{span}_{\mathrm{k}}\left\{\Delta_{1}^{1}, \Delta_{2}^{1}\right\},
$$

where $\Delta_{1}^{1}(1)=g \otimes 1+1 \otimes g$, and $\Delta_{2}^{1}(1)=g \otimes g+1 \otimes 1$.

$$
\mathcal{E}_{A_{2}}=\operatorname{span}_{\mathbb{k}}\left\{\Delta_{1}^{2}, \Delta_{2}^{2}, \Delta_{3}^{2}\right\},
$$

where $\Delta_{1}^{2}(1)=h \otimes 1+h^{2} \otimes h^{2}+1 \otimes h, \Delta_{2}^{2}(1)=h^{2} \otimes 1+h \otimes h+1 \otimes h^{2}$, and $\Delta_{3}^{2}(1)=$ $h \otimes h^{2}+1 \otimes 1+h^{2} \otimes h$. Therefor

$$
\mathcal{E}_{B}=\operatorname{span}_{\mathbb{k}}\left\{\Delta_{1}, \Delta_{2}, \Delta_{3}, \Delta_{4}, \Delta_{5}\right\},
$$

where $\Delta_{1}(1,1)=\left(\Delta_{1}^{1}(1), 0\right), \Delta_{2}(1,1)=\left(\Delta_{2}^{1}(1), 0\right), \Delta_{3}(1,1)=\left(0, \Delta_{1}^{2}(1)\right), \Delta_{4}(1,1)=$ $\left(0, \Delta_{2}^{2}(1)\right)$ and $\Delta_{5}(1,1)=\left(0, \Delta_{3}^{2}(1)\right)$.

Then, the general expression of any nearly Frobenius coproduct in the unit is

$$
\Delta(1,1)=\left(a_{1} \Delta_{1}^{1}\left(1_{G}\right)+a_{2} \Delta_{2}^{1}\left(1_{G}\right), b_{1} \Delta_{1}^{2}\left(1_{H}\right)+b_{2} \Delta_{2}^{2}\left(1_{H}\right)+b_{3} \Delta_{3}^{2}\left(1_{H}\right)\right),
$$

where $a_{i}, b_{j} \in \mathbb{k}$ for $i=1,2$ and $j=1,2,3$.

2. Consider the linear quiver $Q: \underset{1}{\stackrel{\eta}{\longrightarrow}} \stackrel{\bullet}{2}$ and its associated path algebra:

$$
A=\mathbb{k} Q=\left\langle e_{1}, e_{2}, \eta\right\rangle .
$$

It is known that $\mathcal{E}_{A}$ is a vector space of dimension 1 , and a generator is the coproduct $\Delta: A \rightarrow A \otimes A$ defined as

$$
\Delta(1)=\eta \otimes e_{1}+e_{2} \otimes \eta .
$$


Now we will construct the tensor product of two copies of $A$ :

$$
B=A \otimes A=\left\langle e_{1} \otimes e_{1}, e_{1} \otimes e_{2}, e_{2} \otimes e_{1}, e_{2} \otimes e_{2}, e_{1} \otimes \eta, \eta \otimes e_{1}, \eta \otimes e_{2}, e_{2} \otimes \eta, \eta \otimes \eta\right\rangle .
$$

This algebra admits only one coproduct, and it is

$$
\bar{\Delta}=(1 \otimes \tau \otimes 1) \circ(\Delta \otimes \Delta) .
$$

On the other hand, if we consider the next quiver

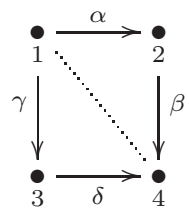

and the algebra $C=\frac{\mathbb{k} Q}{\langle\alpha \beta-\gamma \delta\rangle}=\left\langle\mathbf{e}_{\mathbf{1}}, \mathbf{e}_{\mathbf{2}}, \mathbf{e}_{\mathbf{3}}, \mathbf{e}_{\mathbf{4}}, \alpha, \beta, \gamma, \Delta, \alpha \beta\right\rangle$ we can prove that this algebra is isomorphic to $B$. The isomorphism given on the basis is as follows:

$$
\begin{gathered}
\varphi: B \rightarrow C \\
\varphi\left(e_{1} \otimes e_{1}\right)=\mathbf{e}_{1}, \quad \varphi\left(e_{1} \otimes e_{2}\right)=\mathbf{e}_{2}, \quad \varphi\left(e_{2} \otimes e_{1}\right)=\mathbf{e}_{3}, \quad \varphi\left(e_{2} \otimes e_{2}\right)=\mathbf{e}_{4} \\
\varphi\left(e_{1} \otimes \eta\right)=\alpha, \quad \varphi\left(\eta \otimes e_{2}\right)=\beta, \quad \varphi\left(\eta \otimes e_{1}\right)=\gamma, \quad \varphi\left(e_{2} \otimes \eta\right)=\delta \\
\varphi(\eta \otimes \eta)=\alpha \beta .
\end{gathered}
$$

It is clear that the isomorphism $\varphi$ respects the algebra structures. Then, we can conclude that $\mathcal{E}_{C}$ has dimension one and a generator is:

$$
\Delta(\mathbf{1})=\alpha \beta \otimes \mathbf{e}_{\mathbf{1}}+\beta \otimes \alpha+\delta \otimes \gamma+\mathbf{e}_{\mathbf{4}} \otimes \alpha \beta .
$$

Remark 12. In these lines we want to make notice that we cannot establish a nice property that relates the Frobenius dimension of a quotient algebra with the original algebra. First, in the Example 7 of 2 a nontrivial coproduct is constructed in the quotient algebra $A / J$ from a nontrivial structure on $A$, but $\operatorname{Frobdim}(A)=1$ and $\operatorname{Frobdim}(A / J)=3$.

In addition, we can not always recover a nontrivial structure on the quotient from one on the original algebra, for example if we consider $A=\mathbb{k} \mathbb{A}_{4}=\left\langle e_{1}, e_{2}, e_{3}, e_{4}, \alpha, \beta, \gamma\right\rangle$ with all the arrows having the same orientation and the radical square zero algebra $B=\mathbb{k}_{4} / I$, we know that $A$ admits only one nontrivial nearly Frobenius coproduct, that is

$$
\Delta\left(e_{1}\right)=\alpha \beta \gamma \otimes e_{1}, \Delta\left(e_{2}\right)=\beta \gamma \otimes \alpha, \Delta\left(e_{3}\right)=\gamma \otimes \alpha \beta, \Delta\left(e_{4}\right)=e_{4} \otimes \alpha \beta \gamma,
$$

and this structure is trivial in $B$. But we can prove that $B$ admits nontrivial nearly Frobenius coproducts, moreover Frobdim $B=5$.

In the next paragraph we will give a nice interpretation of the Frobenius space of an algebra $A$ using hochschild cohomology.

Remark 13. For an $A$-bimodule $M$, where $A$ is an algebra we call

$$
M^{A}=\{m \in M: a m=m a \quad \forall a \in A\}
$$


the sub-bimodule of invariants. In Remark 1 of [2] is shown that every nearly coproduct is determined by its value in 1 , that is, we have a linear isomorphism

$$
\Phi: \mathcal{E}_{A} \rightarrow(A \otimes A)^{A} \text {, such that } \Phi(\Delta)=\Delta(1) .
$$

Moreover, if we remember that the 0-group of Hochschild cohomology of $M$ with coefficients in $A$ is

$$
H^{0}(A, M)=\{m \in M: a m=m a \forall a \in A\} .
$$

In particular for $M=A \otimes A$ we have

$$
\mathcal{E}_{A} \cong(A \otimes A)^{A}=H^{0}(A, A \otimes A) .
$$

Then, it is possible to identify the Frobenius space of $A$ with the 0-group of Hochschild cohomology of $A \otimes A$ with coefficients in $A$.

\subsection{Normalized nearly Frobenius algebras}

In the following results we will restrict ourselves to work with a subfamily of nearly Frobenius algebras. This construction is motivated by the notion of normalized Fourier transform (see [5]).

Definition 14. Let $A$ be an algebra and $\Delta$ a nearly Frobenius coproduct, we say that $\Delta$ is normalized if $m \circ \Delta=\operatorname{Id}_{A}$, where $m$ is the product of $A$. If $A$ admits a normalized Frobenius coproduct we will say that $(A, \Delta)$ is a normalized nearly Frobenius algebra.

Example 2.1. Let $G$ be a cyclic finite group. The group algebra $A=\mathbb{k} G$ is a nearly Frobenius algebra. We can consider the nearly Frobenius coproduct $\Delta(1)=\frac{1}{|G|} \sum_{k=1}^{n} g^{k} \otimes g^{n-k}$. It is a simple verification that $\Delta$ is normalized.

Proposition 15. 1. If $A$ and $B$ are nearly Frobenius algebras with normalized coproducts, then $C=A \times B$ has normalized coproduct.

2. If $A$ and $B$ are nearly Frobenius algebras with normalized coproducts, then $D=A \otimes B$ has normalized coproduct.

Proof. By previous results we know that $C$ and $D$ are nearly Frobenius algebras. We only need to prove that the induced coproducts are normalized.

1. In the first case the coproduct is defined as

$$
\Delta(c)=\sum\left(c_{1} a_{1}, 0\right) \otimes\left(a_{2}, 0\right)+\sum\left(0, c_{2} b_{1}\right) \otimes\left(0, b_{2}\right)
$$

where $c=\left(c_{1}, c_{2}\right) \in C$ and $\Delta_{A}\left(1_{A}\right)=\sum a_{1} \otimes a_{2}, \Delta_{B}\left(1_{B}\right)=\sum b_{1} \otimes b_{2}$. Then

$$
m \circ \Delta(c)=\sum\left(c_{1} a_{1} a_{2}, 0\right)+\sum\left(0, c_{2} b_{1} b_{2}\right)
$$

Using that $\Delta_{A}$ and $\Delta_{B}$ are normalized we have that $\sum c_{1} a_{1} a_{2}=c_{1}$ and $\sum c_{2} b_{1} b_{2}=c_{2}$, therefore $m \circ \Delta(c)=\left(c_{1}, c_{2}\right)=c$.

2. In the second case the coproduct is defined as

$$
\Delta_{D}:=\tau \circ \Delta_{A} \otimes \Delta_{B}: D=A \otimes B \rightarrow(A \otimes B) \otimes(A \otimes B),
$$


where $\tau:(A \otimes A) \otimes(B \otimes B) \rightarrow(A \otimes B) \otimes(A \otimes B)=D \otimes D$ is the transposition map. With this notation the product in $D$ can be described as

$$
m_{D}=\left(m_{A} \otimes m_{B}\right) \circ \tau^{-1}:(A \otimes B) \otimes(A \otimes B) \rightarrow A \otimes B .
$$

Then

$$
\begin{aligned}
m_{D} \circ \Delta_{D} & =\left(m_{A} \otimes m_{B}\right) \circ \tau^{-1} \circ \tau \circ\left(\Delta_{A} \otimes \Delta_{B}\right) \\
& =\left(m_{A} \otimes m_{B}\right) \circ\left(\Delta_{A} \otimes \Delta_{B}\right) \\
& =\left(m_{A} \circ \Delta_{A}\right) \otimes\left(m_{B} \circ \Delta_{B}\right) \\
& =i d_{A} \otimes i d_{B}=i d_{D} .
\end{aligned}
$$

This concludes the proof that the coproduct of $D$ is normalized.

\subsection{Separable algebras}

In this section, we present known results about separable algebras and we study their relationship with the notion of normalized nearly Frobenius algebras.

A good reference for this section is $[3$.

Definition 16. Let $R$ be a commutative ring. An $R$-algebra $A$ is called separable if the multiplication map

$$
m: A \otimes_{R} A \rightarrow A
$$

has a section $\sigma$ (i.e. $m \circ \sigma=I d_{A}$ ) which is an A-bimodule homomorphism.

Proposition 17. Let $R$ be a commutative ring and let $A$ be a separable $R$-algebra. Given a section $\sigma$ of $m$ which is an A-bimodule homomorphism, set

$e=\sigma(1)$ and write $e=\sum_{i=1}^{n} x_{i} \otimes_{R} y_{i}$ for suitable $n \in \mathbb{N}$ and $x_{i}, y_{i} \in A$ for every $i=1, \ldots, n$. Then we have

(1) $m(e)=1$ i.e. $\quad \sum_{i=1}^{n} x_{i} y_{i}=1$.

(2) $a e=e a$ i.e. $\sum_{i=1}^{n} a x_{i} \otimes_{R} y_{i}=\sum_{i=1}^{n} x_{i} \otimes y_{i}$ a for every $a \in A$.

Definition 18. Let $A$ be an algebra over a commutative ring $R$. An element $e \in A \otimes_{R} A$ is called a separability element for $A$ (over $R$ ) if e fulfills (1) and (2).

Proposition 19. Let $A$ be an algebra over a commutative ring $R$. Then $A$ is a separable $R$-algebra $\Leftrightarrow A \otimes_{R} A$ contains a separability element for $A$ over $R$.

Moreover any separability element of $A$ is an idempotent element of the ring $A \otimes_{R} A^{o p}$.

Proposition 20. Let $A$ be an algebra over a field $\mathbb{k}$. If $A$ is separable over $\mathbb{k}$, then $\operatorname{dim}_{\mathbb{k}}(A)<\infty$.

Theorem 21. Let $A$ be an algebra over a field $\mathbb{k}$ of finite dimension. Then, the following conditions are equivalent.

(1) A has Hochschild cohomological dimension 0.

(2) $A$ is projective as $A^{e}$-module. 
(3) A has a separability element.

Theorem 22. An algebra $A$ admits a normalized nearly Frobenius coproduct if and only if $A$ is separable.

Proof. If $\Delta$ is a normalized nearly Frobenius coproduct then $\Delta(1) \in A \otimes A$ such that $a \Delta(1)=\Delta(1) a$ $\forall a \in A$, and $m(\Delta(1))=1$. Therefore $e=\Delta(1)$ is a separability element thus $A$ is separable.

Conversely, let be $e$ a separability element, first note that $a e=e a$ for all $a \in A$ then it induces a nearly Frobenius coproducto $\Delta$ such that $\Delta(1)=e$.

Finally, the condition $m(e)=1$ say that $m(\Delta(1))=1$ then $m(\Delta(a))=m(a \Delta(1))=$ $a m(\Delta(1))=a 1=a, \forall a \in A$, thus $m \circ \Delta=\operatorname{Id}_{A}$. Therefore $\Delta$ is a normalized nearly Frobenius coproduct.

Remark 23. Note that we prove, in particular, that every separable algebra is a nearly Frobenius algebra. Moreover, the concept of nearly Frobenius coproduct or nearly Frobenius algebra is a weakening of the concept of separable algebra.

Example 2.2. Every Azumaya algebra is nearly Frobenius algebra. Remember that an R-algebra $A$ is said to be an Azumaya $R$-algebra if $A$ is both central and separable over $R$. See [6].

An immediate consequence of Proposition 18, Theorem 21 and Theorem 22 is the following result.

Proposition 24. A admits a normalized nearly Frobenius coproduct if and only if $A$ has Hochschild cohomological dimension 0.

Definition 25. An algebra $A$ over an algebraically closed field $\mathbb{k}$ is called semisimple if $A$ is finite dimensional and every left A-module is projective.

The next result can be proved using Theorem 4.5.7 of [6] together with the theorem of ArtinWedderburn.

Proposition 26. Let $A$ be a separable algebra over a field $\mathbb{k}$, then $A$ is semisimple. If $\mathbb{k}$ is a perfect field then the concepts are equivalents.

Corollary 27. Every normalized nearly Frobenius algebra is semisimple.

Remark 28. Note that we prove, in Corollary 9 , that if $\mathbb{k}$ is algebraically closed then every semisimple algebra over $\mathbb{k}$ is nearly Frobenius. Now, using Proposition 25, we have that every semisimple algebra is separable if $\mathbb{k}$ is perfect. Then, the result of Corollary 9 can be refined in the following way. If $\mathbb{k}$ is a perfect field and $A$ is a $\mathbb{k}$-algebra, then $A$ is a semisimple algebra if and only if $A$ is a normalized nearly Frobenius algebra.

\subsubsection{Applications}

The following results are known, but this paper presents another way of proving them using the previously determined Frobenius structures.

Matrix algebra: If we consider the matrix algebra $M_{n}(\mathbb{k})$, one nearly Frobenius coproduct of this algebra is

$$
\Delta(I)=\frac{1}{n} \sum_{i, k=1}^{n} E_{i k} \otimes E_{k i}
$$

for this coproduct we have $(m \circ \Delta)(I)=I$. Then $M_{n}(\mathbb{k})$ is separable, in particular, if $\mathbb{k}$ is an algebraically closed field, then $M_{n}(\mathbb{k})$ is semisimple. 
Group algebra: In $A=\mathbb{k} G$, where $G$ is a cyclic finite group, we can define the nearly Frobenius coproduct

$$
\Delta(1)=\frac{1}{|G|} \sum_{k=1}^{n} g^{k} \otimes g^{n-k}
$$

Note that

$$
(m \circ \Delta)(1)=\frac{1}{|G|} \sum_{k=1}^{n} g^{k} g^{n-k}=\frac{1}{|G|} \sum_{k=1}^{n} g^{n}=1
$$

Then $A=\mathbb{k} G$ is separable, and semisimple if $\mathbb{k}$ is an algebraically closed field.

Example 2.3. Retaking the example 1 of Examples [2.1, using the Proposition 15, we can see that $B=A_{1} \times A_{2}$ admits a normalized coproduct then it is separable. Remember that $A_{1}=\mathbb{k} G$ and $A_{2}=\mathbb{k} H$, where $G$ and $H$ are cyclic groups of order 2 and 3 respectively.

Truncated polynomial algebra: Let $A=\frac{\mathbb{k}[x]}{\left\langle x^{n+1}\right\rangle}$ be a nearly Frobenius algebra where a basis of the Frobenius space is

$$
\mathcal{E}=\left\{\Delta_{0}, \Delta_{1}, \ldots, \Delta_{n}\right\}
$$

where

$$
\Delta_{k}(1)=\sum_{i+j=n+k} x^{i} \otimes x^{j}, \quad \text { for } k \in\{0, \ldots, n\}
$$

Then, $\Delta=\sum_{k=0}^{n} a_{k} \Delta_{k}$, where $a_{k} \in \mathbb{k}$ for all $k \in\{0, \ldots, n\}$, is a general nearly Frobenius coproduct.

It is easy to prove that there is not a normalized copoduct:

$$
m \circ \Delta(1)=\sum_{k=0}^{n} a_{k} \sum_{i+j=n+k} x^{i} x^{j}=\sum_{k=0}^{n} a_{k} \sum_{i+j=n+k} x^{n+k}=a_{0} \sum_{i+j=n} x^{n}=(n+1) a_{0} x^{n} \neq 1 .
$$

Then $A=\frac{\mathbb{k}[x]}{\left\langle x^{n+1}\right\rangle}$ is not separable, for all $n \geq 1$.

Path algebra: Finally we consider the path algebra generated by the quiver

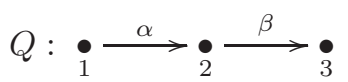

$A=\mathbb{k} Q=\left\langle e_{1}, e_{2}, e_{3}, \alpha, \beta, \alpha \beta\right\rangle . \operatorname{Frobdim}(A)=1$ and the nearly Frobenius coproduct is

$$
\begin{aligned}
& \Delta\left(e_{1}\right)=\alpha \beta \otimes e_{1}, \quad \Delta\left(e_{2}\right)=\beta \otimes \alpha, \quad \Delta\left(e_{3}\right)=e_{3} \otimes \alpha \beta, \\
& \Delta(\alpha)=\alpha \beta \otimes \alpha, \quad \Delta(\beta)=\beta \otimes \alpha \beta, \quad \Delta(\alpha \beta)=\alpha \beta \otimes \alpha \beta
\end{aligned}
$$

Note that $m \circ \Delta\left(e_{1}\right)=\alpha \beta e_{1}=0$, then is not normalized. Therefore $A=\mathbb{k} Q$ is not separable.

\subsection{Bimodule category on normalized nearly Frobenius algebras}

In this section we study the relationship between the normalized nearly Frobenius structure on an algebra and its category of bimodules.

First we give a technical result that will be used later.

Lemma 29. An object $M \in{ }_{A} \mathcal{M}_{A}$ is projective if and only if the structure morphism $\chi: A \otimes M \otimes$ $A \rightarrow M$ splits in ${ }_{A} \mathcal{M}_{A}$. 
Proof. The direct of the assertion is a consequence of the fact that the algebra has unit.

Now we suppose that $\chi: A \otimes M \otimes A \rightarrow M$ splits in ${ }_{A} \mathcal{M}_{A}$, then exists $\nu: M \rightarrow A \otimes M \otimes A$ such that $\chi \circ \nu=\operatorname{Id}_{M}$ in ${ }_{A} \mathcal{M}_{A}$.

As $\nu$ is a homomorphism in ${ }_{A} \mathcal{M}_{A}$ the next diagram commutes

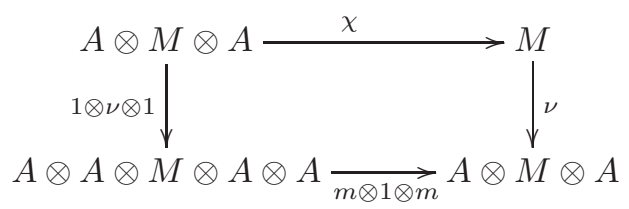

Let be $P, Q \in{ }_{A} \mathcal{M}_{A}, f: M \rightarrow Q$ homomorphism and $g: P \rightarrow Q$ epimorphism in ${ }_{A} \mathcal{M}_{A}$.

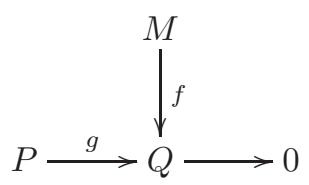

If we only consider the linear structure in the previous diagram we can affirm that there exist a linear map $\alpha: Q \rightarrow P$ such that $g \circ \alpha=\operatorname{Id}_{Q}$. Using this map we define the map $h: M \rightarrow P$ as the composition

$$
M \stackrel{\nu}{\longrightarrow} A \otimes M \otimes A \stackrel{1 \otimes f \otimes 1}{\longrightarrow} A \otimes Q \otimes A \stackrel{1 \otimes \alpha \otimes 1}{\longrightarrow} A \otimes P \otimes A \stackrel{\chi_{P}}{\longrightarrow} P
$$

First we prove that $g \circ h=f$ :

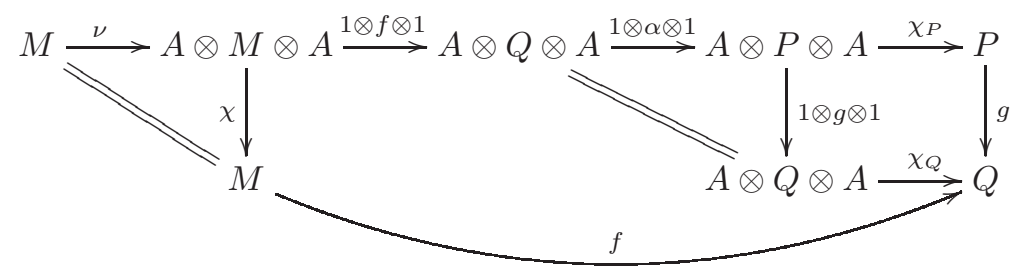

The last step is to prove that $h$ is a homomorphism in ${ }_{A} \mathcal{M}_{A}$, i.e. the diagram

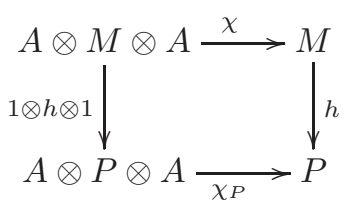

commutes.

Note that $h$ is a composition of homomorphisms in ${ }_{A} \mathcal{M}_{A}$.

$$
h=\chi_{P} \circ(1 \otimes \alpha \otimes 1) \circ(1 \otimes f \otimes 1) \circ \nu .
$$

Let be $A$ a $\mathbb{k}$-algebra and $m: A \otimes A \rightarrow A$ the product of this algebra. Then $\left(A,{ }_{A} m_{A}\right) \in{ }_{A} \mathcal{M}_{A}$ where ${ }_{A} m_{A}: A \otimes A \otimes A \rightarrow A$ is $m(m \otimes 1)=m(1 \otimes m)$.

The following theorem is the central result of this section that allows to relate the normalized nearly Frobenius algebras with their bimodules. 
Theorem 30. The object $\left(A,{ }_{A} m_{A}\right) \in{ }_{A} \mathcal{M}_{A}$ is projective if and only if $A$ admits a normalized nearly Frobenius coproduct.

Proof. If $\left(A,{ }_{A} m_{A}\right)$ is projective bimodule, then, by Lemma 29, there exists $\alpha: A \rightarrow A \otimes A \otimes A$ a homomorphism in ${ }_{A} \mathcal{M}_{A}$ such that ${ }_{A} m_{A} \circ \alpha=\operatorname{Id}_{A}$.

We define $\Delta: A \rightarrow A \otimes A$ as $\Delta=(1 \otimes m) \circ \alpha$.

First note that the normalized condition is immediate:

$$
m \circ \Delta=m \circ(1 \otimes m) \circ \alpha={ }_{A} m_{A} \circ \alpha=\operatorname{Id}_{A} .
$$

To prove that $\Delta$ is an $A$-bimodule homomorphism we need to check that $(m \otimes 1)(1 \otimes \Delta)=\Delta \circ m=$ $(1 \otimes m)(\Delta \otimes 1)$.

Remember that $\alpha$ is a homomorphism in ${ }_{A} \mathcal{M}_{A}$, then the next diagram commutes

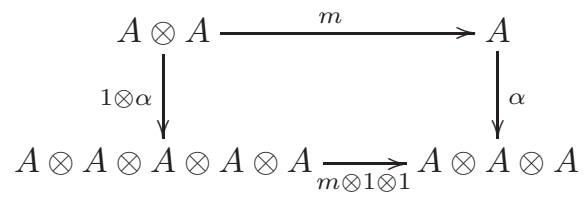

This implies that

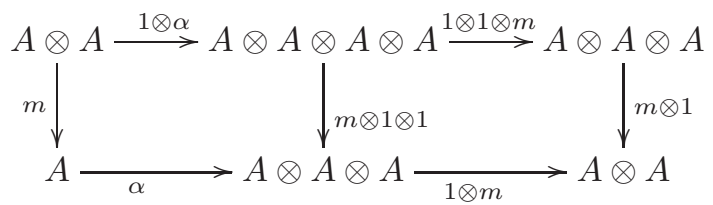

commutes. Then $(m \otimes 1) \circ(1 \otimes \Delta)=\Delta \circ m$.

Applying the associativity of the product and the fact that $\alpha$ is a homomorphism in ${ }_{A} \mathcal{M}_{A}$, in particular in $\mathcal{M}_{A}$, we have that the next diagram commutes

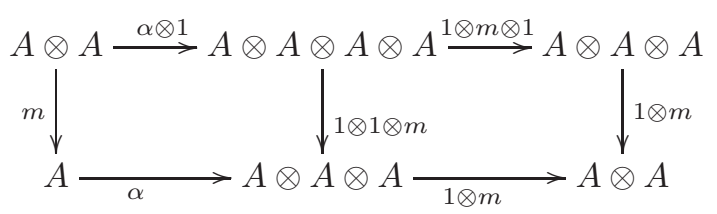

Then $(1 \otimes m) \circ(\Delta \otimes 1)=\Delta \circ m$.

Reciprocally, let be $\Delta: A \rightarrow A \otimes A$ a normalized nearly Frobenius coproduct. Applying the Lemma 29 we need to prove that ${ }_{A} m_{A}$ split, i.e. there exists a morphism $\alpha: A \rightarrow A \otimes A \otimes A$ in ${ }_{A} \mathcal{M}_{A}$ such that ${ }_{A} m_{A} \circ \alpha=\operatorname{Id}_{A}$.

We define $\alpha=(\Delta \otimes 1) \circ \Delta=(1 \otimes \Delta) \circ \Delta$. Note that

$$
{ }_{A} m_{A} \circ \alpha=m \circ(1 \otimes m)(1 \otimes \Delta) \circ \Delta=m \circ(1 \otimes 1) \circ \Delta=m \circ \Delta=\operatorname{Id}_{A} .
$$

To finish we need to prove that $\alpha$ is a homomorphism in ${ }_{A} \mathcal{M}_{A}$, i.e. the next diagram commutes

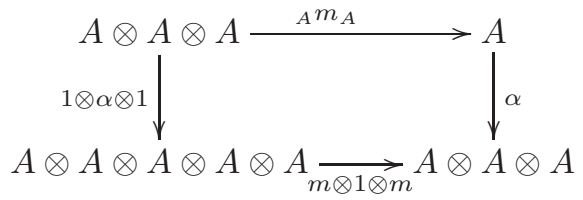




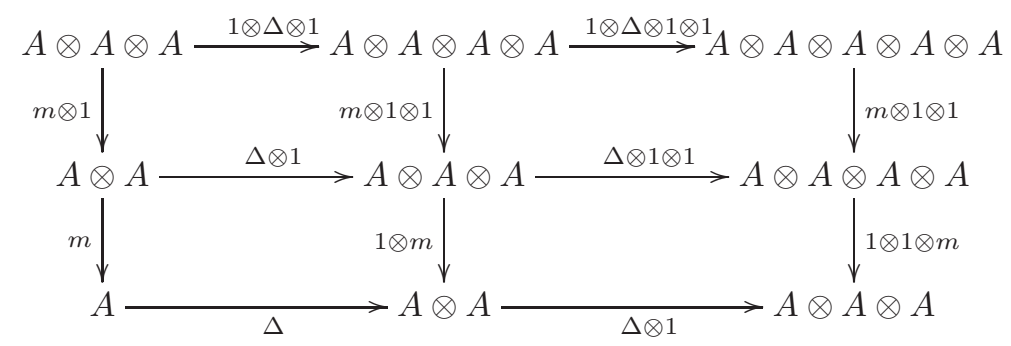

The internal diagrams commute by the nearly Frobenius property of the coproduct $\Delta$. Then the external diagram commutes.

Corollary 31. Let be $A$ a $\mathbb{k}$-algebra. Then, the next conditions are equivalent

(1) A admits a normalized nearly Frobenius algebra.

(2) $\left(A,{ }_{A} m_{A}\right)$ is projective in ${ }_{A} \mathcal{M}_{A}$.

(3) $\operatorname{Every}\left(M, \rho_{M}\right) \in{ }_{A} \mathcal{M}_{A}$ is projective.

(4) The category ${ }_{A} \mathcal{M}_{A}$ is semisimple (every $\left(M, \rho_{M}\right) \in{ }_{A} \mathcal{M}_{A}$ is semisimple).

Proof. (1) $\Leftrightarrow(2)$ is Theorem 30 .

$(3) \Rightarrow(2)$ It is immediate.

$(2) \Rightarrow(3)$ To prove that $\left(M, \rho_{M}\right)$ is projective is equivalent, by the Lemma 29, to prove that $\rho_{M}$ split, i.e. there exists $\alpha_{M}: M \rightarrow A \otimes M \otimes A$ in ${ }_{A} \mathcal{M}_{A}$ such that $\rho_{M} \circ \alpha_{M}=\operatorname{Id}_{M}$.

As $\left(A,{ }_{A} m_{A}\right)$ is projective, by the Theorem 30, there exist $\Delta: A \rightarrow A \otimes A$ nearly Frobenius coproduct normalized. Then we define the map $\alpha_{M}$ as the composition

$$
M \stackrel{u \otimes 1 \otimes u}{\longrightarrow} A \otimes M \otimes A \stackrel{\Delta \otimes 1 \otimes \Delta}{\longrightarrow} A \otimes A \otimes M \otimes A \otimes A \stackrel{1 \otimes \rho_{M} \otimes 1}{\longrightarrow} A \otimes M \otimes A
$$

First we prove that $\rho_{M} \circ \alpha_{M}=\operatorname{Id}_{M}$ :

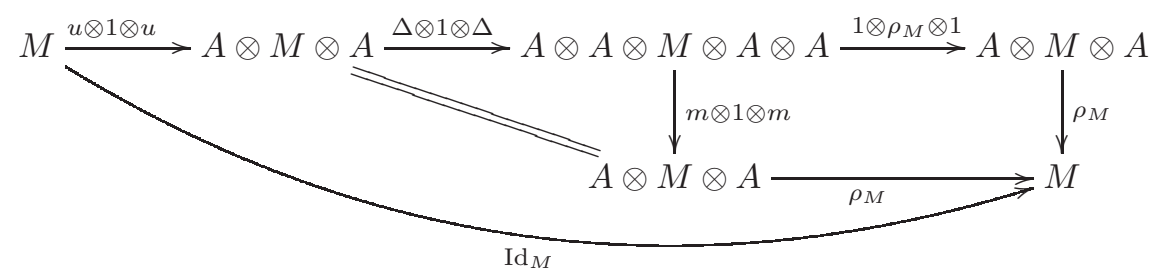

Finally we need to prove that $\alpha_{M}$ is un homomorphism in ${ }_{A} \mathcal{M}_{A}$ i.e. the next diagram commutes

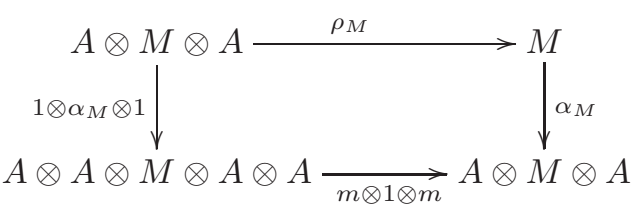




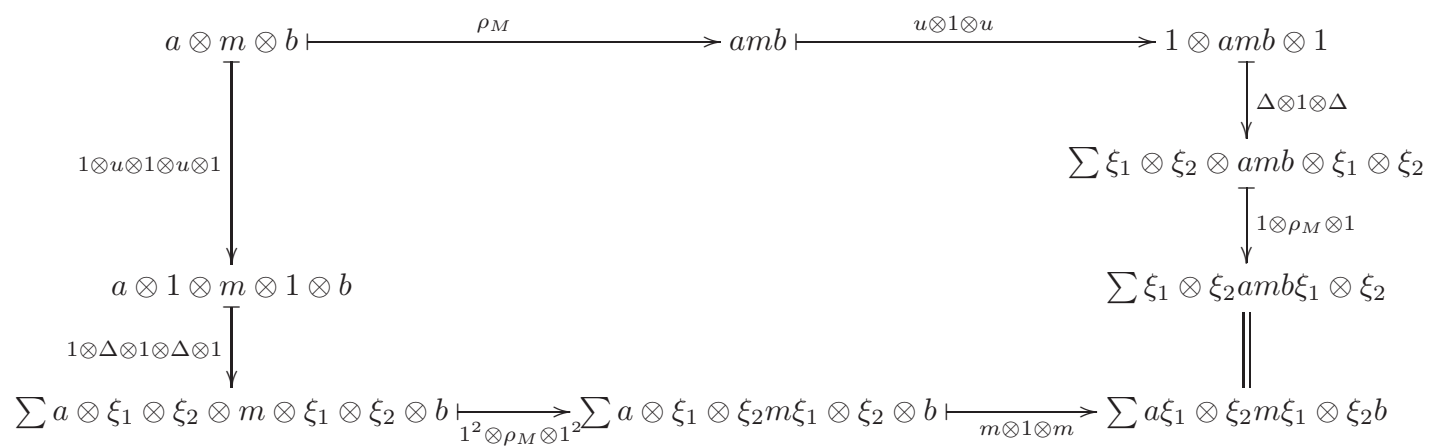

The expressions $\sum a \xi_{1} \otimes \xi_{2} m \xi_{1} \otimes \xi_{2} b$ and $\sum \xi_{1} \otimes \xi_{2} a m b \xi_{1} \otimes \xi_{2}$ agree by the Frobenius condition of the coproduct $\Delta$.

$(3) \Leftrightarrow(4)$ It is a classic result in representation theory (see, for example, [3]).

Remark 32. If we look the examples of section 2.2, we can conclude that the categories of bimodules over $A=M_{n}(\mathbb{k})$ and $B=\mathbb{k} G$ are semisimple, but the category of bimodules over $C=\frac{\mathbb{k}[x]}{\left\langle x^{n+1}\right\rangle}$ is not semisimple.

Finally, with everything developed we relate the studied with the category of modules on a nearly Frobenius algebra.

Theorem 33. If $\mathbb{k}$ is a perfect field we have the following sequence of equivalences: ${ }_{A} \mathcal{M}$ is semisimple $\Leftrightarrow A$ is semisimple $\Leftrightarrow A$ is separable $\Leftrightarrow{ }_{A} \mathcal{M}_{A}$ is semisimple.

If $\mathbb{k}$ is not a perfect field we have the following sequence of implications:

${ }_{A} \mathcal{M}_{A}$ is semisimple $\Leftrightarrow A$ is separable $\Rightarrow A$ is semisimple $\Leftrightarrow{ }_{A} \mathcal{M}$ is semisimple.

\section{References}

[1] H. Abbaspour. On the Hochschild homology of open Frobenius algebras. J. Noncommut. Geom., 10(2) pp. 709-743 (2016).

[2] D. Artenstein, A. González, and M. Lanzilotta, Constructing Nearly Frobenius Algebras. Algebras and Representation Theory (2015) Volume 18, 339-367.

[3] Ibrahim Assem. Algèbres et modules. Les Presses de l’Université d'Ottawa, 1997.

[4] Ralph L. Cohen and Véronique Godin, A polarized view of string topology. Topology, geometry and quantum field theory, London Math. Soc. Lecture Note Ser., vol. 308, Cambridge Univ. Press, Cambridge, 2004, pp. 127-154.

[5] Walter Ferrer Santos, Fourier theory for coalgebras, bicointegrals and injectivity for bicomodules. Hopf Algebras (Lecture Notes in Pure and Applied Mathematics), Volume 237 (2004), 225-240.

[6] Timothy J. Ford, Separable Algebras. Graduate Studies in Mathematics 183. American Mathematical Society Providence, Rhode Island, 2017.

[7] A. González, E. Lupercio, C. Segovia and B. Uribe, Orbifold Topological Quantum Field Theories in Dimension 2. Preprint. 